\title{
Functional demonstrations of starch binding domains present in Ostreococcus tauri starch synthases isoforms
}

\author{
Julieta Barchiesi ${ }^{1}$, Nicolás Hedin ${ }^{1}$, Diego F. Gomez-Casati ${ }^{1}$, Miguel A. Ballicora² and María V. Busi ${ }^{\text {* }}$
}

\begin{abstract}
Background: Starch-binding domains are key modules present in several enzymes involved in polysaccharide metabolism. These non-catalytic modules have already been described as essential for starch-binding and the cataIytic activity of starch synthase III from the higher plant Arabidopsis thaliana. In Ostreococcus tauri, a unicellular green alga of the Prasinophyceae family, there are three SSIII isoforms, known as Ostta SSIII-A, SSIII-B and SSIII-C.

Results: In this work, using in silico and in vitro characterization techniques, we have demonstrated that Ostta SSIIIA, SSIII-B and SSIII-C contain two, three and no starch-binding domains, respectively. Additionally, our phylogenetic analysis has indicated that OsttaSSIII-B, presenting three N-terminal SBDs, is the isoform more closely related to higher plant SSIII. Furthermore, the sequence alignment and homology modeling data gathered showed that both the main 3-D structures of all the modeled domains obtained and the main amino acid residues implicated in starch binding are well conserved in O. tauri SSIII starch-binding domains. In addition, adsorption assays showed that OsttaSSIII-A D2 and SSIII-B D2 domains are the two that make the greatest contribution to amylose and amylopectin binding, while OsttaSSIII-B D1 is also important for starch binding.
\end{abstract}

Conclusions: The results presented here suggest that differences between OsttaSSIII-A and SSIII-B SBDs in the number of and binding of amino acid residues may produce differential affinities for each isoform to polysaccharides. Increasing the knowledge about SBDs may lead to their employment in biomedical and industrial applications.

Keywords: Ostreococcus tauri, Starch-binding domains, Starch synthase, Homology modeling, Adsorption assay

\section{Background}

The generic term carbohydrate binding module (CBM) refers to a contiguous amino acid sequence within a carbohydrate-active enzyme with a distinctive fold presenting carbohydrate-binding activity [1, 2]. Enzymes possessing CBMs share a common modular organization, including a catalytic domain typical for each enzyme and one or more CBMs connected by a loosely-structured chain. CBMs can be found in the middle, $\mathrm{N}$ - or C-terminal positions of the polypeptide chain [3]. They are currently grouped in 71 different families based on their amino acid sequences, substrate binding specificities,

\footnotetext{
*Correspondence: busi@cefobi-conicet.gov.ar

${ }^{1}$ Centro de Estudios Fotosintéticos y Bioquímicos (CEFOBI-CONICET), Universidad Nacional de Rosario, Suipacha 531, 2000 Rosario, Argentina Full list of author information is available at the end of the article
}

location in protein and structures (see http://www.cazy. org/fam/acc_CBM.html) [1]. CBMs from different families are structurally similar. Their ability to bind carbohydrates can be attributed, at least partially, to several aromatic residues which assemble into a hydrophobic surface.

Among CBMs, we can highlight the Starch Binding Domains (SBD), which have acquired the evolutionary advantage of being capable of disrupting the surface of their substrate [4-6]. These domains are distributed in twelve CBM families; 20, 21, 25, 26, 34, 41, 45, 48, 53, 58,68 and 69 [7-10]. The CBM53 family currently has about 96 entries in the CAZy database, representing high phylogenetic diversity, as CBM53 s are found in archaea, bacteria, and eukaryotes. The characterized entries correspond to starch synthase III (SSIII) enzymes belonging 
to higher plant species (Arabidopsis thaliana, Zea mays, Solanum tuberosum, Phaseolus vulgaris, Vignia unguiculata and Oryza sativa) and Chlamydomonas reinhardtii. On the other hand, Ostreococcus tauri SSIII-A and SSIII$\mathrm{B}$ sequences are included in the CBM53 non-characterized entries [11].

Three-dimensional structures are available for nine of the twelve families, except for those classified into CBM45 and CBM53 families. In general, SBD modules comprise $90-130$ residues [12-14], and normally maintain functionality in isolated form [6, 7]. Despite their low sequence similarity, the nine remaining families do share very similar folds, composed by several $\beta$-strands forming an open-sided distorted $\beta$-barrel $[6,7,15,16]$.

An outstanding example of the importance and functionality of an SBD in a plant biosynthetic enzyme is the case of the SSIII isoform from A. thaliana (ArathSSIII), a key regulatory enzyme in starch metabolism, with three SBDs located in tandem in its N-terminal domain, belonging to the CBM53 family [17, 18]. These SBDs, named D1, D2 and D3, have a known regulatory role on ArathSSIII, presenting starch binding activity and also being involved in the modulation of the catalytic properties of the enzyme [17-20].

In the last few years, several nuclear and organellar algae genomes have been sequenced. Some nuclear sequenced genomes from green algae include those from O. tauri [21, 22], O. lucimarinus [23], C. reinhardtii [24], Micromonas pusilla [25], Chlorella variabilis [26] and Volvox carteri [27], among others. C. reinhardtii and $O$. tauri genomes are the best characterized, as documented in numerous publications [21-24]. Although C. reinhardtii has been a model organism for several decades [28], O. tauri has lately gained importance since its first description in 1994 [29].

O. tauri is a picophytoplanktonic species that belongs to the Prasinophyceae, a group of green algae which is thought to have diverged very early from the ancestor of all chloroplast-containing green plants and algae [21, 22]. Although O. tauri constitutes the tiniest eukaryotic cell known and presents the smallest genome of a eukaryotic photosynthetic organism described to date, it contains more SSIII-like genes (three copies) than any other algae and plant species (e.g., A. thaliana presents only one copy) [30]. Thus, the genome of $O$. tauri encodes three SSIIIs, SSIII-A, SSIII-B and SSIII-C, all of which remain uncharacterized to date. The conservation throughout evolution of the three SSIII isoforms, and the absence of SSIV, could be related to the presence of a single starch granule in this alga, which presents similar composition than higher plant starch, but a particular partitioning and propagation mechanism [31].
In summary, this work represents the first characterization of a SBD in algae and based on the results obtained from the in silico analysis, together with the binding profile obtained from the polysaccharide adsorption assays, we postulate that $O$. tauri SSIII-A and SSIII-B proteins possess two and three SBD domains, respectively. In addition, our binding assay results are evidence that both O. tauri SSIII isoforms have different polysaccharide binding specificities.

\section{Results and discussion}

\section{Sequence alignment and phylogenetic analysis of the}

\section{Ostta SSIII SBD domains}

Similarity searches using PSI-Blast [32] retrieved numerous sequences whose GeneBank codes are shown in Additional file 1: Table S1. When sequences corresponding to OsttaSSIII-A and OsttaSSIII-B N-terminal regions, and OsttaSSIII-C entire sequence were used as input, we found sequence similarities with respect to the SSIII from different plants and algae displaying considerably low E-values. In addition, OsttaSSIII-A was very similar $\left(\mathrm{E}=6 \times 10^{-5}\right)$ to a hypothetical protein from Desulfosporosinus meridiei, a bacterium isolated from groundwater contaminated with aromatic compounds derived from fuels [33]. In turn, OsttaSSIII-C also presents high sequence similarity with a hypothetical protein from Parachlamydia acanthamoebae str. Hall's coccus and also with glycogen synthases from Candidatus Protochlamydia amoebophila UWE25 and from cyanobacteriae Anabaena variabilis ATCC 29413, Synechococcus sp. JA3-3Ab and Fischerella sp. JSC-11.

The alignment of the amino acid sequences from OsttaSSIII-A and OsttaSSIII-B SBD regions with homologous sequences identified with PSI-Blast, allowed us to construct a phylogenetic tree for these regions (Fig. 1). The evolutionary relationships deduced from this analysis suggest that OsttaSSIII-A and OsttaSSIII-B SBDs containing regions are most closely related to other alga SSIII proteins rather than to higher plants SSIII, including $A$. thaliana (Fig. 1).

Subsequently, using InterPro [34] and CD-search servers [35], a different number of SBDs were detected in OsttaSSIIIs $\mathrm{N}$-terminal and central protein regions with significantly low E-values (threshold $1 \times 10^{-4}$ ). Three domains were detected in OsttaSSIII-B (D1, D2, and D3, named in order from $\mathrm{N}$-terminal to $\mathrm{C}$-terminal), two in OsttaSSIII-A (D1 and D2) and none in OsttaSSIIIC (Fig. 2A). The five domains share 19-40 \% identity (CLUSTALW [36]). All of them have typical 90-130 aa SBD sizes [13, 14], with the exception of OsttaSSIII-B D1, which is 59 aa long. The analysis of SSIII N-terminal regions from other green algae reveals that $O$. lucimarinus, M. pusilla CCMP1545, M. sp. RCC299 and C. 


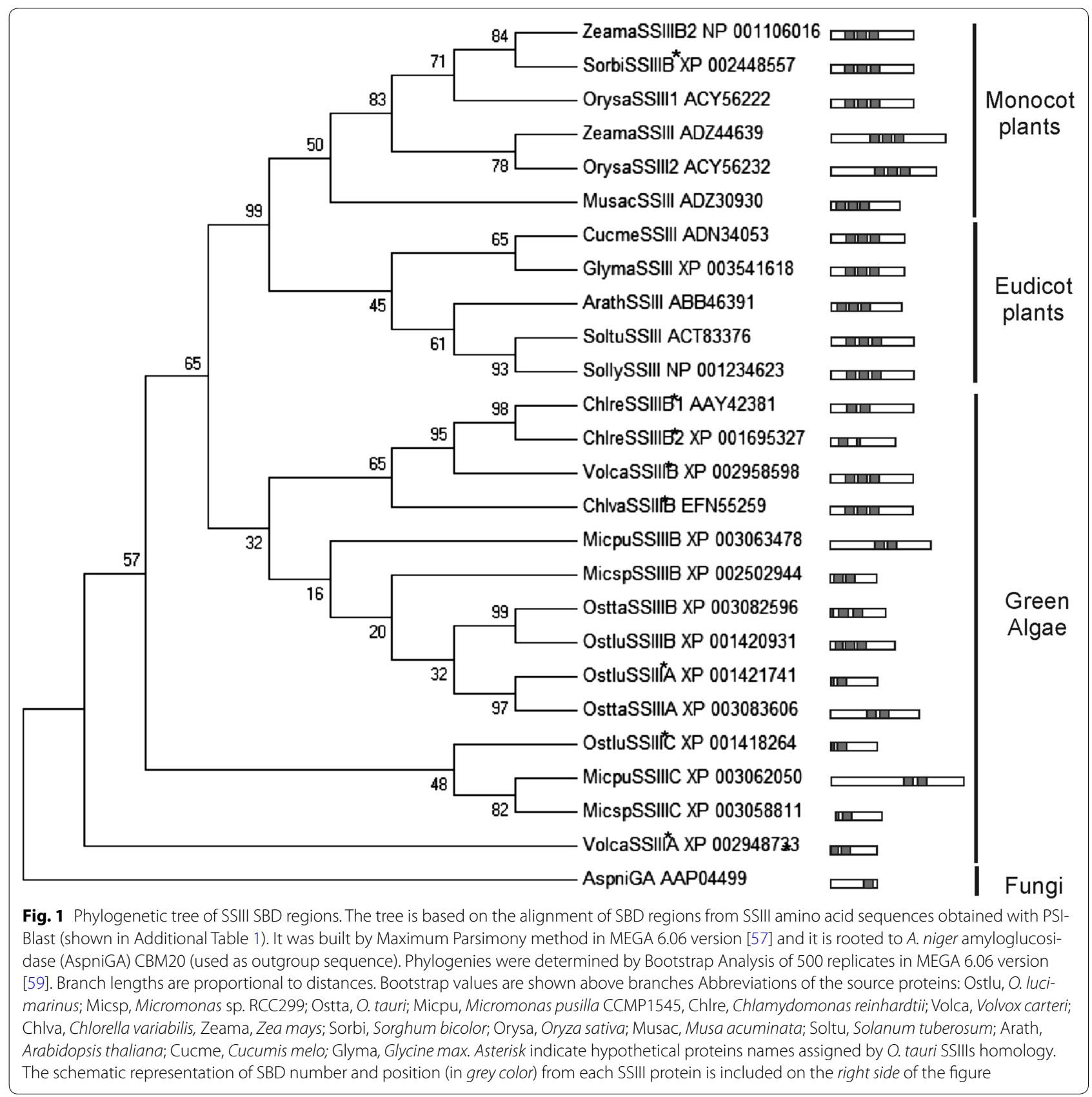

reinhardtii show variable numbers of SBDs in their SSIII isoforms, with some of them exhibiting a reduced size (between 37 and 62 aa) (Fig. 1). These aspects seem to be exclusive of green algae SSIII SBDs, given the fact that all the land plant species with multiple SSIII isoforms analyzed to date present three SBDs with definite size conservation.

Despite the fact that there is no structural information available for the N-terminal D2 domain from ArathSSIII, it is by far one of the best characterized starch binding domains studied. We have previously reported that ArathSSIII D2 contains two binding sites which comprise amino acid residues Y394 (binding site 1) and W366 (binding site 2) acting cooperatively in the binding process with domain D1, while residues G336 and W340 have minor contributions [20]. The alignment of OsttaSSIII and ArathSSIII SBDs sequences shows a high degree of similarity, indicating that these domains are well conserved (Fig. 2B). OsttaSSIII-B D1 and OsttaSSIIIB D2 present higher sequence identity with ArathSSIII 
A

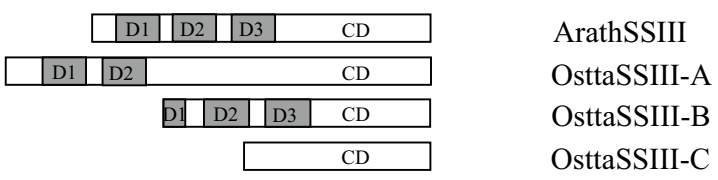

\begin{tabular}{|c|c|c|}
\hline \begin{tabular}{|l|} 
D1 \\
\end{tabular} & & OsttaSSIII-A D1 \\
\hline D2 & & OsttaSSIII-A D2 \\
\hline \begin{tabular}{|l|l|} 
D1 & D2 \\
\end{tabular} & & OsttaSSIII-A D12 \\
\hline & 国 & OsttaSSIII-B D1 \\
\hline & D2 & OsttaSSIII-B D2 \\
\hline & \begin{tabular}{|l|}
$\mathrm{D} 3$ \\
\end{tabular} & OsttaSSIII-B D3 \\
\hline & \begin{tabular}{|l|l|} 
D2 & D3 3 \\
\end{tabular} & OsttaSSIII-B D123 \\
\hline
\end{tabular}

B

ArathSSIII D1 134 -KPDEDIEV-FLNRN-LSTLNNEPDVLIMGAFNEWRWKS------FTRRLEKTWIHE--DWLSCLL 188 ArathSSIII D2 309 -KAEDTVKL-YYNKR-SGPLTNSKELWLHGGFNNWVDGLSIVVKLVNAELKDVDPK-SGNWWFAEV 370 ArathSSIII D3 476 -QAGNPVTV-LYNPA-NTVLNGKPEVWFRGSFNRWTHRLG---PLPPQKMEATDD--ESSHVKTTA 533 OsttaSSIIIAD1 584 ---GSPVTVRYCPN--DRVLHGRES IVMHYGVNDWMG----------ASQVEMKKS-QGGVFIATI 633 OsttaSSIIIAD2 738 ---GQTVKL-YYNPN-NTLLNGSASVFI IGGWNRWSHKE-----KFNVQMAKVTVN-GEVRMQAEL 792 OsttaSSIIIBD1 090 ------------------LR-SASGWKIGGYNAWRVG------EFTKDMIKVK---GTSWVYADV 127 OsttaSSIIIBD2 224 ---GSTERV-MWNKAYNPIGGDTDKLVLHVGFNGWCAGVEAK-FDLVRATGEIKDE-NNDWWVADV 288 OsttaSSIIIBD3 385 ---GKPLKV-FYNKN-NTNLSWTDEIYMTGGFNRWSHEA----AIEPVKMTPPGAG-EEFFTVTVP 440 PaepoAMY CBM25 455 GGTGNKVTI-YYKKGFNS-----PYIHYRPAGGSWTAAP----G-VKMQDAEIS-----GYAKITV 504 BachaAMY CBM25 006 SGDATDITI-YYKTGWTH-----PHIHYSLNQGAWTTLP----G-VPLTKS-----------VKVTI 050 RatnoAMPK CBM48 079 -----PTVFRWTGGGKE--------VYLSGSFNNWS-K-------LPLTRSQ-------NNFVAIL 116 1c

ArathSSIII D1 189 HIPKEAYKMDFVFFNGQ--S---VYDNNDSKDFCVEIKGGMDKVDFENFL------------ 233

ArathSSIII D2 371 WPGGALVIDWVFADGPP-KGAFIYDNNGYQDFHALVPQKLPE------------------ 412

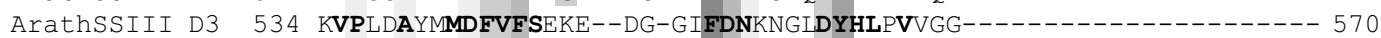

OsttaSSIIIAD1 634 DVPVTALVMDVVFSDGSQ-T----YDNNNKSDFHVPVL------------------------ 666

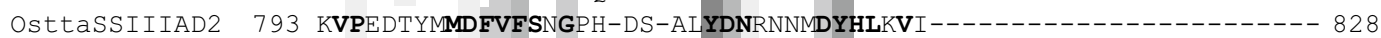

OsttaSSIIIBD1 128 NVDERAFSMDFVEKGDG-----EAYEKDEGQDFSLTV----------------------- 159

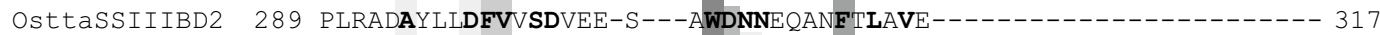

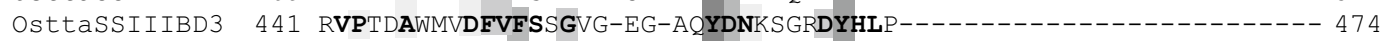

PaepoAMY CBM25 505 DIGSAS-QLEAAFNDGNN-N----WDSNNTKNYSFSTGTSTYTPGNSGNAGTITSGAPAG- 558

BachaAMY CBM25 051 EAEEGS-QLRAAFNNGSG-Q----WDNNQGRDYDFSSGVHTLADG------RILSGTP---- 100

RatnoAMPK CBM48 117 DLPEGEHQYKFFV-DGQ------WT----HDPSEP-IVTSQLGTVNNIIQV---------- 156

Fig. 2 A Schematic representation of ArathSSIII, OsttaSSIIIs and OsttaSSIII recombinant SBDs. CD, catalytic domain. B Alignment between OsttaSSIIIA D1 and D2, OsttaSSIII-B D1, D2 and D3, ArathSSIII D1, D2, and D3, and RatnoAMPK $\beta$-subunit GBD (CBM48), BachaAMY CBM25 and PaepoAMY CBM25 used as the template for homology modeling. 1 and 2 above the alignment indicate binding-site 1 and 2 characterized in $A$. thaliana. 1 a, $1 \mathrm{~b}$ and 1c indicate G335, W340 and Y394, and 2 W366 (all correspond to A. thaliana numbering, [20]), respectively. Other conserved positions are shown in bold and four levels of gray represents $100,90,82,78$ and $55 \%$ of conservation

D2 (25 and $21.2 \%$ identity, respectively), than what is observed with ArathSSIII D1 and D3. Meanwhile, OsttaSSIII-A D1, OsttaSSIII-A D2 and OsttaSSIII-B D3 amino acid sequences are more similar to ArathSSIII D3 (29.5, 40 and $43 \%$ identity, respectively), than to ArathSSIII D1 and D2.

Furthermore, the amino acid residues involved in ArathSSIII D2 starch binding are highly conserved in OsttaSSIII SBDs. There is high conservation of residues G335, W340 and Y394 [the first one is exchanged by a tyrosine residue in OsttaSSIII-A D1 and by a valine residue in OsttaSSIII-B D2, and the last one is exchanged by a tryptophan residue in OsttaSSIII-B D2 (Fig. 2B)].
On the other hand, W366, proposed to be essential in ArathSSIII D2 binding site 2 for the binding to amylose, amylopectin and starch, is only conserved in OsttaSSIIIB D2. This residue was replaced by a phenylalanine in OsttaSSIII-A D1, and by a methionine, a valine and a threonine in OsttaSSIII-A D2, OsttaSSIII-B D1 and OsttaSSIII-B D3, respectively. Our results suggest that the second polysaccharide binding site seems to be exclusive of OsttaSSIII-A D1 and OsttaSSIII-B D2 domains. However, the neighboring residue W365 is conserved in OsttaSSIII-B D1 and OsttaSSIII-B D2. Further investigation is necessary to analyze its possible role in polysaccharide binding. 


\section{Molecular model of the OsttaSSIII SBDs}

The homology models of the OsttaSSIII SBDs based on CBM25-1 of beta/alpha-amylase from Paenibacillus polymyxa (PDB code: 2LAA), CBM25 from Bacillus halodurans amylase (PDB code: $2 \mathrm{C} 3 \mathrm{~V}$ ) and Rattus novergicus glycogen-binding (GBD) domain of the AMP-activated protein (PDB code: 1Z0N) [37-39], are shown in Fig. 3b. To obtain the homology models, we used the three templates in parallel (Fig. 3a), using the best part of each template with the Modeller program. Regardless of the low identity with these templates (10-23\% for OsttaSSIII-A D1, OsttaSSIII-A D2, OsttaSSIII-B D2 and OsttaSSIII-B D3), the four OsttaSSIII SBDs were predicted by threading to have a similar $\beta$-sandwich fold. The confidence of the prediction using GenTHREADER $[40,41]$ was high, with the $p$ value lower than $10^{-3}$ for $2 \mathrm{C} 3 \mathrm{~V}$ and $2 \mathrm{LAA}$ templates. Although $1 \mathrm{ZON}$ template p-value was around $10^{-2}$, it was also included because of the high score obtained for it in a previous fold class assignation with @TOME V2 [42]. On the other hand, OsttaSSIII-B D1 identity with the templates was inferior (9-11\%), mainly due to its short amino acid sequence, and the confidence of the prediction using GenTHREADER was low. However, OsttaSSIII-B D1 model was also generated with the same three templates (PDB: $1 \mathrm{ZON}, 2 \mathrm{C} 3 \mathrm{~V}$ and $2 \mathrm{LAA}$ ) as a first approximation to estimate a short starch binding domain fold.

It is important to note that the templates may have similar roles to the domains modeled. AMPK is an AMP-activated protein kinase responsible for the coordination of cellular metabolism in response to energy demands and many other stimuli. AMPK- $\beta$ subunit acts as a platform for the $\alpha$-catalytic and $\gamma$-regulatory subunits and targets the AMPK heterotrimer to glycogen [39, $43,44]$. The AMPK- $\beta$ glycogen binding domain belongs to the CBM48 family, and presents a $24-25 \%$ sequence identity with OsttaSSIII-A D1, OsttaSSIII-A D2, OsttaSSIII-B D2 and OsttaSSIII-B D3. The family is mainly represented by CBMs appended to GH13 subfamily [45] and the beta subunit (glycogen-binding) of mammalian AMP-activated protein kinases (AMPK) [39]. It is worth mentioning that there is a clear evolutionary relatedness of the CBM48, CBM53 and CBM20 families, which suggests the existence of a common clan hosting most of the known SBDs [7]. Moreover, the alignment of the amino acid sequences of CBM20, CBM21, CBM48 and CBM53 illustrates the close evolutionary relationship of the four CBM families and reveals only subtle differences in the polysaccharide-binding sites, showing a high degree of conservation [7].

Instead, $P$. polymyxa amylase and $B$. halodurans alpha/beta amylase, both extracellular bacterial starch degrading enzymes, present starch binding domains belonging to CBM25 family. The modules from this family are frequently linked to amylolytic enzymes from the family GH13, but there are CBM25 examples found in families GH14, GH31 and GH119 [9, 46]. With regard to $B$. halodurans amylase, it contains both CBM26 and CBM25. Each SBD binds starch individually, though the affinity for both modules together was 50 times higher $[9,37]$.

Based on the results obtained with the validation tools Verify 3D and Rampage, we are able to state that our models exhibit good quality, but most importantly, certainly suggests a strong structural similarity among these domains and their templates considering the level of sequence identity observed.

All the SDB models created show a similar fold to that described for RatnoAMPK- $\beta$ GBD and BachaAMY CBM25 with a compact $\beta$ sandwich structure composed of two antiparallel $\beta$-sheets (Fig. 3). One $\beta$-sheet from AMPK- $\beta$ GBD is formed by strands $\beta 1$ and $\beta 4$, and the other $\beta$-sheet contains strands $\beta 2, \beta 3, \beta 5$ and $\beta 6$ plus a $\beta$-hairpin $\beta 7-\beta 8$. The AMPK- $\beta$ GBD structure presents a carbohydrate binding pocket with residues W100, W133, K126, L146, G147, T148 and N150 (1Z0N numbering) involved in polysaccharide binding [39, 43, 47]. Residues W100 and W133 correspond to W340 and Y394 (ArathSSIII D2 numbering), implicated in starch binding in ArathSSIII D2 [20]. The BachaAMY CBM25 structure in complex with maltotetraose shows a polysaccharide binding site formed by the residues W34, W74, H26, D81 and N76 (2C3V numbering). BachaAMY CBM25 has only one main maltooligosaccharide binding site [37]. Residues W34 and W74 correspond to W340 and Y394 involved in starch binding in ArathSSIII D2, as mentioned above [20].

Despite the definite conservation of the main 3-D structures of all the modeled domains, there are some minor differences. None of them have the $\beta 7-\beta 8$ hairpin loop present in RatnoAMPK- $\beta$ GBD at the C-terminal end. OsttaSSIII-A D1 and OsttaSSIII-A D2 also lack the $\beta 3$ strand. Absence of the $\beta 3$ strand is a common feature of CGTases [48] and maltogenic $\alpha$-amylases [7, 49]. Besides, the $\beta$-hairpin $\beta 7-\beta 8$ is missing in previously identified folding of the $\mathrm{E}$ domain of a cyclodextrin glycosyltransferase and the $\mathrm{C}$-terminal domain of a $\beta$-amylase from both Bacillus cereus and Aspergillus niger glucoamylases [39]. Further investigation is necessary to analyze the implications of the differences among the OsttaSSIII SBDs structures that ultimately affect their polysaccharide binding specificities and biological functions.

A comparison of the RatnoAMPK- $\beta$ GBD subunit, BachaAMY CBM25 and PaepoAMY CBM25 with those from OsttaSSIII SBDs showed a $100 \%$ conservation of the W100/W34 residue (1Z0N/2C3V numbering) in all 
a

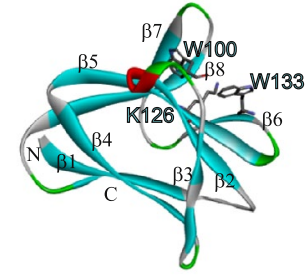

RatnoAMPK- $\beta$

(1ZON)

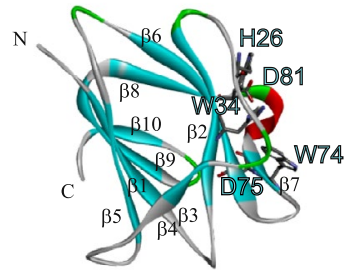

BachaCBM25

(2C3V)

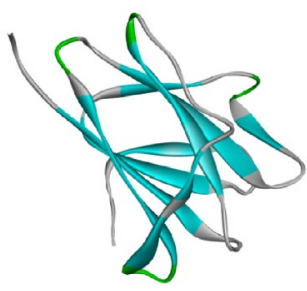

PaepoCBM25

(2LAA)

b
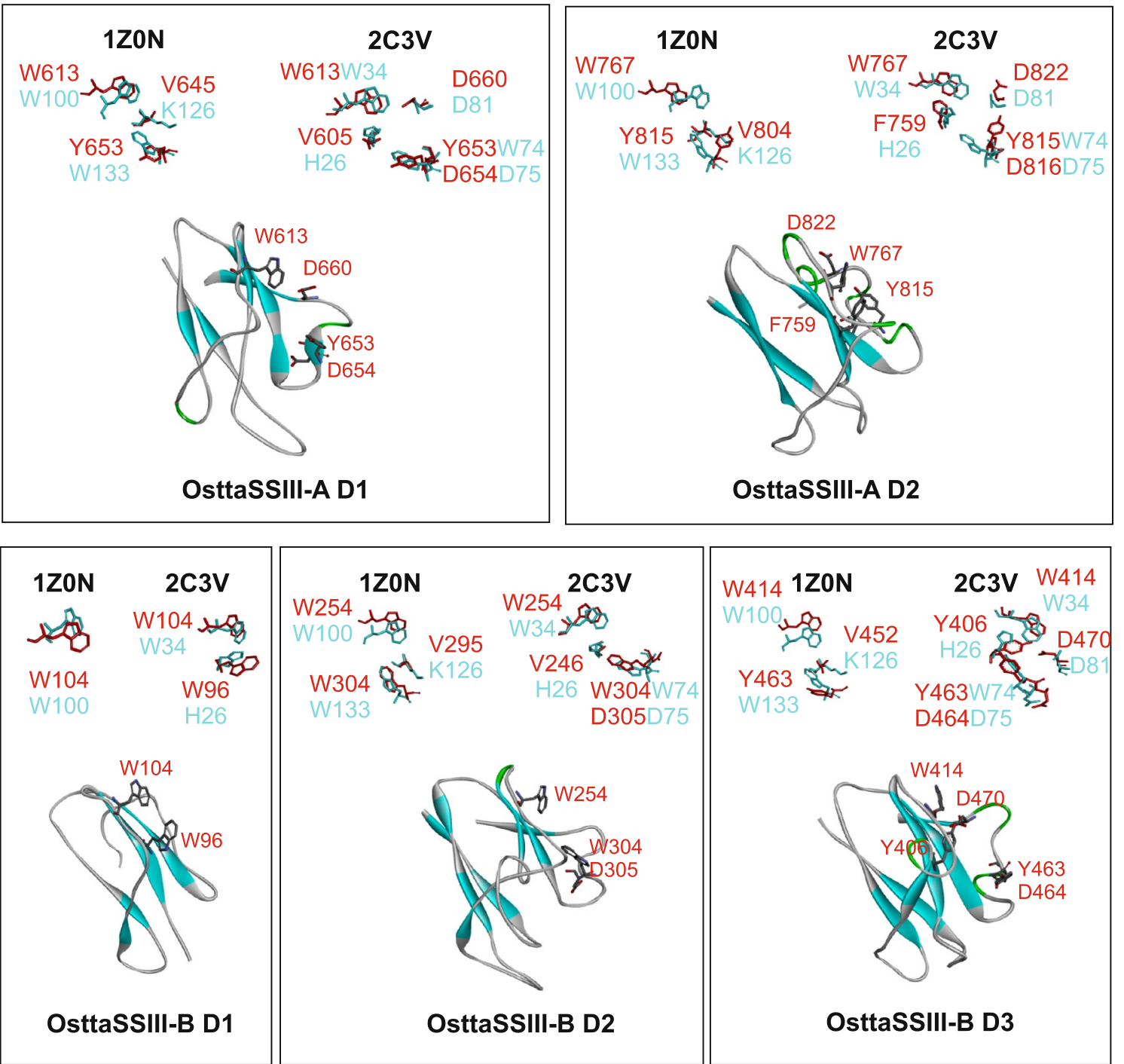

Fig. 3 Homology modeling of OsttaSSIIls SBDs. a Structural model of Rattus norvegicus AMPK $\beta$-subunit GBD (RatnoAMPK- $\beta$, PDB code: 1 ZON), Bacillus halodurans amylase CBM25 (BachaCBM25, PDB code: 2C3V) and Paenibacillus polymyxa amylase CBM25 (PaepoCBM25, PDB code: 2LAA); binding residues are shown. b Proposed models for OsttaSSIII-A D1, OsttaSSIII-A D2, OsttaSSIII-B D1, OsttaSSIII-B D2 and OsttaSSIII-B D3; putative binding residues are shown. Superimposition of OsttaSSIII-A D1, OsttaSSIII-A D2, OsttaSSIII-B D1, OsttaSSIII-B D2 and OsttaSSIII-B D3 binding residues (red) and 1 ZON or 2C3V (cyan) binding residues, is shown next to each model 
sequences (Fig. 2B). Besides, these residues have a conserved position in the five models proposed (Fig. 3b). Instead, the W133/W74 residue $(1 \mathrm{Z0N} / 2 \mathrm{C} 3 \mathrm{~V}$ numbering) is conserved in OsttaSSIII-B D2 sequences, but is replaced by tyrosine in the other four analyzed sequences. Moreover, these residues do present a similar spatial location in the models, with exception of OsttaSSIII-B D1 which lacks an aromatic amino acid in this spatial region. Regarding the spatial position of the basic residue $\mathrm{K} 126$ (1Z0N numbering), it was replaced by a valine residue in OsttaSSIII-A D1, -A D2, -B D2 and -B D3, and is absent in the OsttaSSIII-B D1 model structure. This particular amino acid replacement in the binding site could be a consequence of architectural disparities between starch and glycogen. On the other hand, a conservation of an aromatic or aliphatic side chain suggests the importance of a possible hydrophobic interaction at this point.

We also noted a superposition of the aspartic residue at D75 (2C3V PDB numbering) in the OsttaSSIII-A D1, -A D2, -B D2 and -B D3 models. Besides, the spatial location of residue $\mathrm{D} 81$ (2C3V numbering) is conserved in OsttaSSIII-A D1, -A D2 and -B D3, and it is replaced by phenylalanine in OsttaSSIII-B D2. On the other hand, the $\mathrm{H} 26$ residue (2C3V numbering) is replaced by aromatic residues such as phenylalanine (in OsttaSSIII-A D2), tryptophan (in OsttaSSIII-B D1) and tyrosine (in OsttaSSIII-B D3). The role that these residues might play in the starch binding activity of these domains is analyzed below.

\section{OsttaSSIII SBDs polysaccharide binding assays}

Carbohydrate binding modules are frequently associated with carbohydrate active enzymes, specifically those that are active on polysaccharides. They have several functions: align the substrates with the reaction centers, disrupt the surface of the substrates and provide support for macromolecular aggregates. However, the presence of CBMs is not universal and is in fact rare among some families of enzymes [50]. There are examples of enzymes that are active on polysaccharides and have no CBMs in structure. Their catalytic domains contain residues that are responsible for the binding of the carbohydrate to the enzyme, but distant from the active site. These residues form a surface site or a secondary binding surface (SBS). The functions of the SBSs are not limited to the orientation of the enzyme to the substrate, but also include the substrate guidance to the active site and allosteric site, altering the specificity of the enzyme. Within families of GH13 and GH77 about 45 enzymes with one SBS were identified. SBSs and CBMs usually co-occur in enzymes and they probably have complementary functions [50].
The different number of SBDs in OsttaSSIII isoforms may be indicative of different functional characteristics. The binding efficiency of individual or tandem recombinant purified OsttaSIII SBDs (see Fig. 2A) to insoluble polysaccharides was evaluated. We analyzed their ability to bind starch, amylose and amylopectin in co-sedimentation and adsorption assays.

As shown in Additional file 2: Figure S1, all individual OsttaSSIII SBD were found in the pellet with amylose and amylopectin at different levels, except OsttaSIII-A D1. This domain lacks the amino acid residues equivalents to G335 and W365, which are involved in polysaccharide binding in ArathSSIII D2 (Fig. 2) [20]. In tandem OsttaSIII-A D12 bound remarkably to amylose, probably as a result of different amino acids exposed to the polysaccharide and also by the presence of a cooperative effect between OsttaSIII-A D1 and OsttaSIII-A D2. On the other hand, OsttaSIII-A D12 did not bind to amylopectin. Its higher molecular mass could be a steric impediment against this branched substrate. Under the assayed conditions, starch binding capacity was observed only in OsttaSIII-B D1 and OsttaSIII-B D123 (Additional file 2: Figure S1). Consistently, when Aspergillus niger amyloglucosidase was used as a positive binding control, it showed higher co-sedimentation towards amylose rather than starch.

To further characterize the polysaccharide binding affinity, we determined the adsorption constant $\left(K_{\mathrm{ad}}\right)$ in an adsorption assay, at different protein concentration as was previously described [19]. Figure 4 shows the adsorption isotherms for the binding of OsttaSSIII-A D12 and OsttaSSIII-B D123 to starch, amylose and amylopectin.

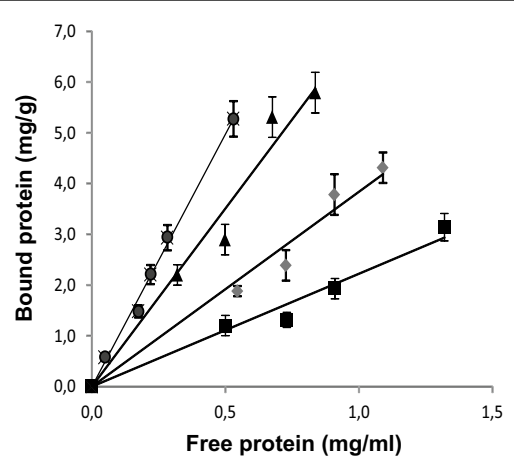

Fig. 4 Adsorption of purified OsttaSSIII SBD proteins to polysaccharides: OsttaSSIII-A D12 with amylose (filled circle),OsttaSSIII-B D123 with amylose (filled triangle),OsttaSSIII-B D123 with amylopectin (filled diamond), and OsttaSSIII-B D123 with starch (filled square). Linear adsorption isotherms indicate the apparent equilibrium distribution of OsttaSSIII SBD proteins between the solid (bound protein) and liquid phase (free protein) at different protein concentrations. $K_{\mathrm{ad}}$ (milliliters per gram of polysaccharide) values represent the slopes of each isotherm 
Table 1 presents OsttaSSIII SBDs determined $K_{\text {ad }}$ values [19].

Consistently with co-sedimentation assay, all recombinants OsttaSSIII SBD showed amylose and amylopectin affinity, except OsttaSIII-A D1 (Table 1). In tandem OsttaSIII-A D12 presents higher amylose affinity, and starch binding capacity was observed only in OsttaSIIIB D1 and OsttaSIII-B D123, and in lower levels with respect to amylose.

The OsttaSIII-B D1 domain binds to all the polysaccharides assayed, despite its small size. This 59 amino acid residue module conserves all starch binding amino acids described for ArathSSIII D2, with the exception of W366; however, W365 and/or Y367 could compensate for its absence, probably as an alternative binding site. As already mentioned, it is important to highlight that OsttaSSIII-B D1 presents higher sequence identity with ArathSSIII D2 than what is observed with ArathSSIII D1 and D3. In addition, the aromatic residue W96 (OsttaSSIII D1 numbering) is in a similar spatial position than the residue $\mathrm{H} 26$ (2C3V numbering) suggesting a role in polysaccharide binding (Fig. 3b), however, this remains to be determined. These results suggest that the different SBD organization in OsttaSIII isoforms could provide precise polysaccharide affinities, reinforcing the evolutionary conservation of the isoforms. It stands to reason that additional investigation will be required to test this premise.

In summary, we have found that O. tauri SSIII-A and SSIII-B isoforms present two and three SBD, respectively. This inference is based on fold class assignment results, sequence alignment data, the analysis of the conservation of specific residues implicated in starch binding and the results obtained from the polysaccharide binding assays. The statistical evaluation of the models obtained using independent methods, as Rampage evaluation criteria and the Verify-3D structure evaluation server, gives further support to the assignment of the OsttaSSIII SBDs folds

Table 1 Adsorption constants for OsttaSSIII SBD recombinant proteins

\begin{tabular}{llll}
\hline Isoform & \multicolumn{2}{l}{$\boldsymbol{K}_{\mathbf{a d}}(\mathbf{m L} / \mathbf{g})$} & \\
\cline { 2 - 4 } & Starch & Amylose & Amylopectin \\
\hline OsttaSSIII-A D1 & ND & ND & ND \\
OsttaSSIII-A D2 & ND & $7.32 \pm 0.53$ & $7.50 \pm 0.65$ \\
OsttaSSIII-A D12 & ND & $9.95 \pm 0.82$ & ND \\
OsttaSSIII-B D1 & $3.70 \pm 0.43$ & $7.13 \pm 0.61$ & $3.12 \pm 0.34$ \\
OsttaSSIII-B D2 & ND & $7.23 \pm 0.42$ & $8.22 \pm 0.59$ \\
OsttaSSIII-B D3 & ND & $1.84 \pm 0.20$ & $3.12 \pm 0.28$ \\
OsttaSSIII-B D123 & $2.22 \pm 0.21$ & $7.02 \pm 0.44$ & $3.84 \pm 0.31$ \\
\hline
\end{tabular}

to CBM family 25, 48 and 53 such as ArathSSIII D1, D2, and D3. The construction of a phylogenetic tree allowed us to determine that OsttaSSIII-A and OsttaSSIII-B SBD containing regions are evolutionarily closer to other green algae SSIIIs rather than the enzymes from higher plants. Additionally, we observed variable SBD sizes and amounts in green algae SSIII enzymes. Therefore, our results suggests that $O$. tauri possesses three SSIII isoforms, one similar to higher plants SSIII, presenting three N-terminal SBDs (OsttaSSIII-B) and the other two isoforms, one with two central SBDs (OsttaSSIII-A) and the third lacking SBDs (OsttaSSIII-C). The biological significance of the last two should be further studied.

Most of the residues responsible for binding to glycogen in Agrobacterium tumefaciens GlgA are conserved in the catalytic domain of ArathSSIII [51] and OsttaSSIII (Additional file 3: Figure S2). Four of seven residues analyzed and listed in Busi et al. 2008 [51] are present in OsttaSSIII-C (E25, Y112, D146 and R270, OsttaSSIII-C numbering) and may constitute an SBS. We could infer that the difference in the mechanism of action of the three OsttaSSIII proteins was mainly due to the combined action of SBDs and SBSs in OsttaSSIII-A and OsttaSSIII-B and only SBSs in OsttaSSIII-C.

SBSs and SBDs have been studied in great detail beginning a few years ago. Researchers have developed strategies for the identification and characterization of these sites, using techniques that measure their binding properties as well as looking at the influence on enzymatic activity of altering these sites through mutagenesis. Our group has recently published the optimization of the kinetic parameters of a GlgA from bacteria by adding one, two or three plant SBDs which have the capacity to bind to polysaccharides. Our results also showed that the chimeric enzyme has an increased capacity to synthesize glycogen in vivo [52].

This growing interest to study SBDs may eventually lead to applications involving them in industrial and biomedical settings [53-55]. These modules provide an interesting way to regulate enzymatic activity without altering the structure of the active site of the enzyme. Due to OsttaSSIII SBDs ability to bind to starch, amylose and amylopectin, we propose the use of these modules as biotechnological tools for modifying the quality and/ or amount of starch in plants and algae. In conclusion, our results constitute an important cluster of information concerning evolutionary and structure-function aspects of SBD domains.

\section{Methods}

\section{Homology modeling and sequence alignment}

The sequence similarity searches of OsttaSSIII-A (Genbank code: XP_003083606, 1-843 aa residues) N-terminal

ND no determined 
region, OsttaSSIII-B (Genbank code: XP_003082596, 1-386 aa residues) $\mathrm{N}$-terminal region and OsttaSSIII-C (Genbank: XP_003079955) whole amino acid sequence were performed with PSI-BLAST [32] with the default parameters (inclusion threshold 0.005) until convergence, using no redundant databases. Sequences with significant matches to the query sequence (threshold expectation value ("E value") below $1 \times 10^{-4}$ ) were retrieved and aligned with the program CLUSTALW [36]. Alignment of the OsttaSSIII-B N-terminal amino acid sequences with homologous sequences were performed by using the Unipro UGENE v.1.10.4 program [56] with default parameters. Alignment of the OsttaSSIII, ArathSSIII, AgrtuGS and EsccoGS catalytic domain (CD) amino acid sequences were performed by using the Unipro UGENE v.1.10.4 program [56] with default parameters. Phylogenetic SSIII SBD regions tree was built by Maximum Parsimony method in MEGA 6.06 version [57]. The tree was rooted by $A$. niger amyloglucosidase CBM20. Phylogenies were determined by Bootstrap Analysis of 500 replicates in MEGA 6.06 version.

We have used two approaches to detect domains in OsttaSSIIIs N-terminal region: the CD-search server [35] and the InterPRO resource [34]. SBDs were aligned with the program CLUSTALW [36] and manually improved with the program Bioedit [58].

Threading and fold recognition were achieved using the program GenTHREADER [40, 41]. Homology modeling of OsttaSSIIIs SBD domains was performed using the program Modeller 9.13 [59]. Each structure was modeled using three templates; CBM25-1 of beta/alpha-amylase from Paenibacillus polymyxa (PDB code 2LAA), CBM25 from Bacillus halodurans amylase (PDB code 2C3V) and Rattus novergicus glycogen-binding domain of the AMPactivated protein complexed with beta-cyclodextrin (PDB code 1Z0N) [37-39, 47]. Alignment with the templates was based on homology and secondary structure. The reliability of the model was evaluated using the programs Rampage (a structure validation tool for the assessment of the Ramachandran plot of proteins) [60] and Verify3D [61]. Superimposition of structures was performed using the MultiProt server [62].

\section{Cloning, expression and purification of OsttaSSIII SBD}

Starch binding domains OsttaSSIII-A D1, D2 and tandem D12 (Ot16g01560) were cloned from O. tauri genomic DNA (kindly provide for Dr. Evelyne Derelle) into EcoRI and HindIII sites of pRSET-B (Invitrogen CA, USA). Starch binding domains OsttaSSIII-B D1, D2, D3 and tandem D123 were cloned from O. tauri genomic DNA (Ot13g01250) into KpnI and HindIII sites of pRSET-C (Invitrogen CA, USA), using standard molecular biology procedures and the primers:
AD1 Fw, CCAGAATTCGGATCACCCGTC; AD1 Rev, CGAAAGCTTGAGAACGGGAACATG; AD2 Fw, TCGAATTCGGGCAGACTGTCAAG; AD2 Rev, CGAAAGCTTTGAGGTGATAATCC; BD1 Fw, TGGGTACCCATCGGTGGATACAACGCG, BD1 Rev, GCGAAGCTTCGAACTAGACAGTC; BD2 Fw, GGGGTACCCGGGAGCACGGAGCG; BD2 Rev, CTTAAGCTTTTACTCCACTGCGAGCG; BD3 Fw, CCGGTACCTGGCAAACCGCTCAAG; and BD3 Rev, GCGAAGCTTCCTCGCTAGGGGAG. These expression vectors were transformed in Escherichia coli BL21 (DE3) pLysS strain. Cells were grown at $37^{\circ} \mathrm{C}$ for $3 \mathrm{~h}$, $1 \mathrm{mM}$ IPTG was added and incubated at $30^{\circ} \mathrm{C}$ for at least $4 \mathrm{~h}$. Cells were harvested by centrifugation at $5000 \times \mathrm{g}$ for 15 min at $4{ }^{\circ} \mathrm{C}$. Each pellet was suspended in buffer containing $20 \mathrm{mM}$ Tris- $\mathrm{HCl}$ (pH 7.5). Cells were disrupted by sonication and centrifuged at $12000 \times g$ for $15 \mathrm{~min}$ at $4{ }^{\circ} \mathrm{C}$. The homogenates were loaded onto a HiTrap chelating HP column (GE Healthcare BioSciences, Uppsala, Sweden) equilibrated with binding buffer $[20 \mathrm{mM}$ Tris- $\mathrm{HCl}(\mathrm{pH} 7.5)$ and $20 \mathrm{mM}$ imidazole]. The column was washed with 10-15 volumes of binding buffer, and each protein was eluted using a linear gradient of binding buffer and elution buffer $[20 \mathrm{mM}$ Tris- $\mathrm{HCl}(\mathrm{pH} 7.5)$ and 20-500 mM imidazole] [18]. The presence of the SBD in elution fractions was monitored by SDS-PAGE analysis. Obtained SBD sizes: OsttaSSIII-A D1 16 kDa, OsttaSSIII-A D2 15 kDa, tandem OsttaSSIII-A D12 34 kDa, OsttaSSIII-B D1 8 kDa, OsttaSSIII-B D2 $11 \mathrm{kDa}$, OsttaSSIII-B D $310 \mathrm{kDa}$, tandem OsttaSSIII-B D123 $48 \mathrm{kDa}$. The protein containing fractions were concentrated to $>1 \mathrm{mg} / \mathrm{mL}$ using Vivaspin $63000 \mathrm{MWCO}$ concentrators (GE Healthcare BioSciences, Uppsala, Sweden), desalted and stored at $4{ }^{\circ} \mathrm{C}$ until they were used.

\section{Polysaccharide-binding assays}

Purified SBD above described (approximately $20 \mu \mathrm{g}$ each) were mixed with starch (Fluka-85649, St. Louis, MO, USA), amylose (Fluka-10130, St. Louis, MO, USA) or amylopectin (Fluka10118, St. Louis, MO, USA) in $20 \mathrm{mM}$ Tris- $\mathrm{HCl}(\mathrm{pH} 7.5)$, at a final polysaccharide concentrations of $10 \%(\mathrm{w} / \mathrm{v})$. A control was done without polysaccharide. Binding was done at room temperature by orbital mixing for $30 \mathrm{~min}$. Polysaccharides were obtained by centrifugation at $12000 \times g$ for $5 \mathrm{~min}$, and the supernatant removed and boiled in SDS loading buffer. The pellets were washed three times with $100 \mu \mathrm{L} 20 \mathrm{mM}$ Tris- $\mathrm{HCl}(\mathrm{pH} 7.5)$ by gentle vortexing and centrifugation, and then they were boiled in SDS loading buffer in a final volume equal to the supernatants for SDS-PAGE [63]. Protein levels were determined by densitometry of SDSPAGE gels that had been stained with $0.25 \%$ Coomassie Blue R-250 in $45 \% \mathrm{v} / \mathrm{v}$ methanol, $10 \% \mathrm{v} / \mathrm{v}$ acetic acid, 
then destained in $25 \% \mathrm{v} / \mathrm{v}$ methanol, $7 \% \mathrm{v} / \mathrm{v}$ acetic acid, using a scanner and Adobe Photoshop (Adobe Systems Inc., Mountain View, CA). Arabidopsis thaliana frataxin was used as negative control [64].

The adsorption constant (Kad) of SBD was measured as described previously with minor modifications $[18,19]$. Purified recombinant SBD proteins (final concentration of $0-80 \mu \mathrm{M}$ ) were mixed with starch (Fluka-85649, St. Louis, MO, USA), amylose (Fluka-10130, St. Louis, MO, USA) or amylopectin (Fluka10118, St. Louis, MO, USA) in $20 \mathrm{mM}$ Tris- $\mathrm{HCl}(\mathrm{pH} 7.5)$, at a final polysaccharide concentrations of $10 \%(\mathrm{w} / \mathrm{v})$. A control was done without polysaccharide. Binding was done at room temperature by orbital mixing for $30 \mathrm{~min}$ and centrifuged at $12000 \times g$ for $5 \mathrm{~min}$ at $4{ }^{\circ} \mathrm{C}$. To calculate the amount of bound protein, the amount of protein in the supernatant was subtracted from the total protein added to the mixture. The protein concentration was determined by Lowry method. The adsorption constant (Kad, in milliliters per gram of polysaccharide) was determined from the slope as previously reported $[18,19]$.

\section{Additional files}

Additional file 1: Table S1. Sequences Retrieved from Similarity Searches.

Additional file 2: Figure S1. OsttaSSIII SBD-polysaccharide COsedimentation assay. A. OsttaSSIlls SBDs were incubated with starch, amylopectin, or amylose and examined for their ability to co-sediment. Then, they were localized to the supernatant $(S)$ or pellet $(P)$ fraction in SDS-PAGE, as described in Methods section. B. Densitometric quantifications of OsttaSSIII SBD-polysaccharide co-sedimentation assay presented in A. Polysaccharide bound or free protein levels are shown. The density for supernatant fraction of buffer incubated samples (control) was set to $100 \%$ for each experiment, and density for pellet fraction of buffer samples was consider basal sedimentation and subtracted from the other pellet fractions in each experiment. Data correspond to mean values of at least three independent experiments. Error bars correspond to standard deviations (*, $P<0.001$ ). AspniGA: Aspergillus niger glucoamylase (positive control), ArathFt: Arabidopsis thaliana frataxin (negative control). OsttaSSIIIA D1 16 kDa, OsttaSSIII-A D2 15 kDa, OsttaSSIII-A D12 34 kDa, OsttaSSIII-B D1 8 kDa, OsttaSSIII-B D2 11 kDa, OsttaSSIII-B D 310 kDa, OsttaSSIII-B D123 $48 \mathrm{kDa}$, ArathFt $15 \mathrm{kDa}$, and AspniGA $66 \mathrm{kDa}$.

Additional file 3: Figure S2. Alignment between, ArathSSIII-CD, OsttaSSIII-CD, AgrtuGS CD and EsccoGS CD. Conserved positions, corresponding to glycogen binding sites in ArathSSIII CD, are shown in bold and shaded in grey. CD: catalytic domain; Arath: Arabidopsis thaliana: AgrtuGS: Agrobacterium tumefaciens glycogen synthase; Escco: Escherichia coli glycogen synthase.

\section{Authors' contributions}

$J B, D G C, M A B$ and $M V B$ designed the conception and delineation of the study; and prepared the manuscript and reviewed it before submission. JB performed the in silico characterization. JB and $\mathrm{NH}$ conducted the required experiments, performed the acquisition of the data or analyzed such information. All authors read and approved the final manuscript.

\section{Author details}

${ }^{1}$ Centro de Estudios Fotosintéticos y Bioquímicos (CEFOBI-CONICET), Universidad Nacional de Rosario, Suipacha 531, 2000 Rosario, Argentina. ${ }^{2}$ Department of Chemistry and Biochemistry, Loyola University Chicago, 405 Flanner Hall, 1068 W Sheridan Road, Chicago, IL 60660, USA.

\section{Acknowledgements}

We thank Lic. Celeste Buchensky for the generous gift of the ArathFt purified protein and Lic. Mauricio Grisolía for helpful discussion of the in silico analysis. We are grateful to Dr. Mariana Martín and Dr. Celina Galles for helpful discussions and critical reading of the manuscript. We thank Dr. Evelyne Derelle for kindly providing O. tauri genomic DNA. This work was supported by Grants from Consejo Nacional de Investigaciones Científicas y Técnicas (CONICET, PIP 00134) and Agencia Nacional de Promoción Científica y Tecnológica (ANPCyT, PICT RAICES 2011-0982; PICT Joven 2012-0981). JB, MVB and DGC are research members from CONICET. MAB is assistant chair from Loyola University. NH is a doctoral fellow from ANPCYT.

\section{Competing interests}

The authors declare that they have no competing interests.

Received: 8 June 2015 Accepted: 19 October 2015

Published online: 28 October 2015

\section{References}

1. Boraston AB, Bolam DN, Gilbert HJ, Davies GJ. Carbohydrate-binding modules: fine-tuning polysaccharide recognition. Biochem J. 2004:382(Pt 3):769-81.

2. Guillén D, Sánchez S, Rodríguez-Sanoja R. Carbohydrate-binding domains: multiplicity of biological roles. Appl Microbiol Biotechnol. 2010;85(5):1241-9.

3. Shoseyov O, Shani Z, Levy I. Carbohydrate binding modules: biochemical properties and novel applications. Microbiol Mol Biol Rev. 2006;70(2):283-95.

4. Penninga D, van der Veen BA, Knegtel RM, van Hijum SA, Rozeboom $\mathrm{HJ}$, Kalk KH, Dijkstra BW, Dijkhuizen L. The raw starch binding domain of cyclodextrin glycosyltransferase from Bacillus circulans strain 251. J Biol Chem. 1996:271(51):32777-84.

5. Williamson MP, Le Gal-Coeffet MF, Sorimachi K, Furniss CS, Archer DB, Williamson G. Function of conserved tryptophans in the Aspergillus niger glucoamylase 1 starch binding domain. Biochemistry. 1997;36(24):7535-9.

6. Giardina T, Gunning AP, Juge N, Faulds CB, Furniss CS, Svensson B, Morris VJ, Williamson G. Both binding sites of the starch-binding domain of Aspergillus niger glucoamylase are essential for inducing a conformational change in amylose. J Mol Biol. 2001;313(5):1149-59.

7. Christiansen C, Abou Hachem M, Janecek S, Vikso-Nielsen A, Blennow A, Svensson B. The carbohydrate-binding module family 20-diversity, structure, and function. FEBS J. 2009;276(18):5006-29.

8. Cameron EA, Kwiatkowski KJ, Lee BH, Hamaker BR, Koropatkin NM, Martens EC. Multifunctional nutrient-binding proteins adapt human symbiotic bacteria for glycan competition in the gut by separately promoting enhanced sensing and catalysis. mBio. 2014;5(5):e01414-41.

9. Majzlova K, Janecek S. Two structurally related starch-binding domain families CBM25 and CBM26. Biologia. 2014;69(9):1087-96.

10. Peng H, Zheng Y, Chen M, Wang Y, Xiao Y, Gao Y. A starch-binding domain identified in alpha-amylase (AmyP) represents a new family of carbohydrate-binding modules that contribute to enzymatic hydrolysis of soluble starch. FEBS Lett. 2014;588(7):1161-7.

11. Cantarel BL, Coutinho PM, Rancurel C, Bernard T, Lombard V, Henrissat B. The carbohydrate-active enzymes database (CAZy): an expert resource for glycogenomics. Nucleic Acids Res. 2009;37(Database issue):D233-8.

12. Machovic M, Svensson B, MacGregor EA, Janecek S. A new clan of CBM families based on bioinformatics of starch-binding domains from families CBM20 and CBM21. FEBS J. 2005;272(21):5497-513.

13. Rodriguez-Sanoja R, Oviedo N, Sanchez S. Microbial starch-binding domain. Curr Opin Microbiol. 2005;8(3):260-7.

14. Machovic M, Janecek $S$. The evolution of putative starch-binding domains. FEBS Lett. 2006;580(27):6349-56.

15. Lawson CL, van Montfort R, Strokopytov B, Rozeboom HJ, Kalk KH, de Vries GE, Penninga D, Dijkhuizen L, Dijkstra BW. Nucleotide sequence and X-ray structure of cyclodextrin glycosyltransferase from Bacillus 
circulans strain 251 in a maltose-dependent crystal form. J Mol Biol. 1994;236(2):590-600.

16. Sorimachi K, Le Gal-Coeffet MF, Williamson G, Archer DB, Williamson MP. Solution structure of the granular starch binding domain of Aspergillus niger glucoamylase bound to beta-cyclodextrin. Structure. 1997;5(5):647-61.

17. Palopoli N, Busi MV, Fornasari MS, Gomez-Casati D, Ugalde R, Parisi G. Starch-synthase III family encodes a tandem of three starch-binding domains. Proteins. 2006;65(1):27-31.

18. Valdez HA, Busi MV, Wayllace NZ, Parisi G, Ugalde RA, Gomez-Casati DF Role of the $\mathrm{N}$-terminal starch-binding domains in the kinetic properties of starch synthase III from Arabidopsis thaliana. Biochemistry. 2008:47(9):3026-32

19. Wayllace NZ, Valdez HA, Ugalde RA, Busi MV, Gomez-Casati DF. The starch-binding capacity of the noncatalytic SBD2 region and the interaction between the $\mathrm{N}$ - and $\mathrm{C}$-terminal domains are involved in the modulation of the activity of starch synthase III from Arabidopsis thaliana. FEBS J. 2010;277(2):428-40

20. Valdez HA, Peralta DA, Wayllace NZ, Grisolía MJ, Gomez-Casati DF, Busi MV. Preferential binding of SBD from Arabidopsis thaliana SSIII to polysaccharides: study of amino acid residues involved. Starch Stärke. 2011;63(8):451-60.

21. Derelle E, Ferraz C, Rombauts S, Rouze P, Worden AZ, Robbens S, Partensky F, Degroeve S, Echeynie S, Cooke R, et al. Genome analysis of the smallest free-living eukaryote Ostreococcus tauri unveils many unique features. Proc Natl Acad Sci USA. 2006;103(31):11647-52.

22. Blanc-Mathieu R, Verhelst B, Derelle E, Rombauts S, Bouget FY, Carre I, Chateau A, Eyre-Walker A, Grimsley N, Moreau H, et al. An improved genome of the model marine alga Ostreococcus tauri unfolds by assessing Illumina de novo assemblies. BMC Genom. 2014;15:1103.

23. Palenik B, Grimwood J, Aerts A, Rouze P, Salamov A, Putnam N, Dupont C, Jorgensen R, Derelle E, Rombauts S, et al. The tiny eukaryote Ostreococcus provides genomic insights into the paradox of plankton speciation. Proc Natl Acad Sci USA. 2007;104(18):7705-10.

24. Merchant SS, Prochnik SE, Vallon O, Harris EH, Karpowicz SJ, Witman GB, Terry A, Salamov A, Fritz-Laylin LK, Marechal-Drouard L, et al. The Chlamydomonas genome reveals the evolution of key animal and plant functions. Science. 2007;318(5848):245-50.

25. Worden AZ, Lee JH, MockT, Rouze P, Simmons MP, Aerts AL, Allen AE, Cuvelier ML, Derelle E, Everett MV, et al. Green evolution and dynamic adaptations revealed by genomes of the marine picoeukaryotes Micromonas. Science. 2009;324(5924):268-72.

26. Blanc G, Duncan G, Agarkova I, Borodovsky M, Gurnon J, Kuo A, Lindquist E, Lucas S, Pangilinan J, Polle J, et al. The Chlorella variabilis NC64A genome reveals adaptation to photosymbiosis, coevolution with viruses, and cryptic sex. Plant Cell. 2010;22(9):2943-55.

27. Prochnik SE, Umen J, Nedelcu AM, Hallmann A, Miller SM, Nishii I, Ferris P, Kuo A, Mitros T, Fritz-Laylin LK, et al. Genomic analysis of organismal complexity in the multicellular green alga Volvox carteri. Science. 2010;329(5988):223-6

28. Harris EH. Chlamydomonas as a model organism. Annu Rev Plant Physiol Plant Mol Biol. 2001;52:363-406.

29. Courties CVA, Troussellier M, Lautier J, Chretiennot-Dinet MJ, Neveux J, Machado C, Claustre H. Smallest eukaryotic organism. Nature. 1994;370:1.

30. Deschamps P, Moreau H, Worden AZ, Dauvillee D, Ball SG. Early gene duplication within chloroplastida and its correspondence with relocation of starch metabolism to chloroplasts. Genetics. 2008;178(4):2373-87.

31. Ral JP, Derelle E, Ferraz C, Wattebled F, Farinas B, Corellou F, Buleon A Slomianny MC, Delvalle D, d'Hulst C, et al. Starch division and partitioning. A mechanism for granule propagation and maintenance in the picophytoplanktonic green alga Ostreococcus tauri. Plant Physiol. 2004;136(2):3333-40.

32. Altschul SF, Madden TL, Schaffer AA, Zhang J, Zhang Z, Miller W, Lipman DJ. Gapped BLAST and PSI-BLAST: a new generation of protein database search programs. Nucleic Acids Res. 1997;25(17):3389-402.

33. Robertson WJ, Bowman JP, Franzmann PD, Mee BJ. Desulfosporosinus meridiei sp. nov., a spore-forming sulfate-reducing bacterium isolated from gasolene-contaminated groundwater. Int J Syst Evol Microbiol. 2001:51(Pt 1):133-40.

34. Hunter S, Jones P, Mitchell A, Apweiler R, Attwood TK, Bateman A, Bernard T, Binns D, Bork P, Burge S, et al. InterPro in 2011: new developments in the family and domain prediction database. Nucleic Acids Res. 2012:40(Database issue):D306-12.

35. Marchler-Bauer A, Bryant SH. CD-Search: protein domain annotations on the fly. Nucleic Acids Res. 2004;32(Web server issue):W327-31.

36. Larkin MA, Blackshields G, Brown NP, Chenna R, McGettigan PA, McWilliam H, Valentin F, Wallace IM, Wilm A, Lopez R, et al. Clustal W and Clustal $X$ version 2.0. Bioinformatics. 2007;23(21):2947-8.

37. Boraston AB, Healey M, Klassen J, Ficko-Blean E. Lammerts van Bueren A Law V: a structural and functional analysis of alpha-glucan recognition by family 25 and 26 carbohydrate-binding modules reveals a conserved mode of starch recognition. J Biol Chem. 2006;281(1):587-98.

38. Kawazu T, Nakanishi Y, Uozumi N, Sasaki T, Yamagata H, Tsukagoshi N, Udaka S. Cloning and nucleotide sequence of the gene coding for enzymatically active fragments of the Bacillus polymyxa beta-amylase. J Bacteriol. 1987;169(4):1564-70.

39. Polekhina G, Feil SC, Gupta A, O'Donnell P, Stapleton D, Parker MW. Crystallization of the glycogen-binding domain of the AMP-activated protein kinase beta subunit and preliminary X-ray analysis. Acta Crystallogr Sect F Struct Biol Cryst Commun. 2005;61(Pt 1):39-42.

40. Jones DT. GenTHREADER: an efficient and reliable protein fold recognition method for genomic sequences. J Mol Biol. 1999;287(4):797-815.

41. Lobley A, Sadowski MI, Jones DT. pGenTHREADER and pDomTHREADER: new methods for improved protein fold recognition and superfamily discrimination. Bioinformatics. 2009;25(14):1761-7.

42. Pons JL, Labesse G. @TOME-2: a new pipeline for comparative modeling of protein-ligand complexes. Nucleic Acids Res. 2009;37(Web Server issue):W485-91.

43. McBride A, Ghilagaber S, Nikolaev A, Hardie DG. The glycogen-binding domain on the AMPK beta subunit allows the kinase to act as a glycogen sensor. Cell Metab. 2009;9(1):23-34.

44. Bieri M, Mobbs JI, Koay A, Louey G, Mok YF, Hatters DM, Park JT, Park KH, Neumann D, Stapleton D, et al. AMP-activated protein kinase beta-subunit requires internal motion for optimal carbohydrate binding. Biophys J. 2012;102(2):305-14.

45. Machovič M, Janeček Š. Domain evolution in the GH13 pullulanase subfamily with focus on the carbohydrate-binding module family 48 . Biologia. 2008;63(6):1057-68.

46. Lombard V, Golaconda Ramulu H, Drula E, Coutinho PM, Henrissat B. The carbohydrate-active enzymes database (CAZy) in 2013. Nucleic Acids Res. 2014:42(Database issue):D490-5.

47. Polekhina G, Gupta A, Michell BJ, van Denderen B, Murthy S, Feil SC, Jennings IG, Campbell DJ, Witters LA, Parker MW, et al. AMPK beta subunit targets metabolic stress sensing to glycogen. Curr Biol. 2003;13(10):867-71.

48. Harata K, Haga K, Nakamura A, Aoyagi M, Yamane K. X-ray structure of cyclodextrin glucanotransferase from alkalophilic Bacillus sp. 1011. Comparison of two independent molecules at $1.8 \mathrm{~A}$ resolution. Acta Crystallogr Sect D Biol Crystallogr. 1996;52(Pt 6):1136-45.

49. Dauter Z, Dauter M, Brzozowski AM, Christensen S, Borchert TV, Beier L, Wilson KS, Davies GJ. X-ray structure of Novamyl, the five-domain "maltogenic" alpha-amylase from Bacillus stearothermophilus: maltose and acarbose complexes at 1.7A resolution. Biochemistry. 1999;38(26):8385-92.

50. Cockburn D, Nielsen MM, Christiansen C, Andersen JM, Rannes JB, Blennow A, Svensson B. Surface binding sites in amylase have distinct roles in recognition of starch structure motifs and degradation. Int J Biol Macromol. 2015;75:338-45.

51. Busi MV, Palopoli N, Valdez HA, Fornasari MS, Wayllace NZ, Gomez-Casati DF, Parisi G, Ugalde RA. Functional and structural characterization of the catalytic domain of the starch synthase III from Arabidopsis thaliana. Proteins. 2008;70(1):31-40.

52. Martin M, Wayllace NZ, Valdez HA, Gomez-Casati DF, Busi MV. Improving the glycosyltransferase activity of Agrobacterium tumefaciens glycogen synthase by fusion of N-terminal starch binding domains (SBDs). Biochimie. 2013;95(10):1865-70.

53. Levy I, Paldi T, Shoseyov O. Engineering a bifunctional starch-cellulose cross-bridge protein. Biomaterials. 2004;25(10):1841-9.

54. Machovic M, Janecek S. Starch-binding domains in the post-genome era. Cell Mol Life Sci. 2006;63(23):2710-24.

55. Stephen P, Tseng KL, Liu YN, Lyu PC. Circular permutation of the starchbinding domain: inversion of ligand selectivity with increased affinity. Chem Commun (Camb). 2012;48(20):2612-4. 
56. Okonechnikov K, Golosova O, Fursov M. Unipro UGENE: a unified bioinformatics toolkit. Bioinformatics. 2012;28(8):1166-7.

57. Tamura K, Stecher G, Peterson D, Filipski A, Kumar S. MEGA6: molecular evolutionary genetics analysis version 6.0. Mol Biol Evol. 2013;30(12):2725-9

58. Hall TA. BioEdit: a user-friendly biological sequence alignment editor and analysis program for Windows 95/98/NT. Nucleic Acids Symp Ser. 1999:41:95-8.

59. Sali A. Comparative protein modeling by satisfaction of spatial restraints Mole Med Today. 1995;1(6):270-7.

60. Lovell SC, Davis IW, Arendall WB 3rd, de Bakker PI, Word JM, Prisant MG, Richardson JS, Richardson DC. Structure validation by Calpha geometry: phi, psi and Cbeta deviation. Proteins. 2003;50(3):437-50.
61. Luthy R, Bowie JU, Eisenberg D. Assessment of protein models with threedimensional profiles. Nature. 1992;356(6364):83-5.

62. Shatsky M, Nussinov R, Wolfson HJ. A method for simultaneous alignment of multiple protein structures. Proteins. 2004;56(1):143-56.

63. Kerk D, Conley TR, Rodriguez FA, Tran HT, Nimick M, Muench DG, Moorhead GB. A chloroplast-localized dual-specificity protein phosphatase in Arabidopsis contains a phylogenetically dispersed and ancient carbohydrate-binding domain, which binds the polysaccharide starch. Plant J. 2006;46(3):400-13.

64. Maliandi MV, Busi MV, Clemente M, Zabaleta EJ, Araya A, Gomez-Casati DF. Expression and one-step purification of recombinant Arabidopsis thaliana frataxin homolog (AtFH). Protein Expr Purif. 2007;51(2):157-61.
Submit your next manuscript to BioMed Central and take full advantage of:

- Convenient online submission

- Thorough peer review

- No space constraints or color figure charges

- Immediate publication on acceptance

- Inclusion in PubMed, CAS, Scopus and Google Scholar

- Research which is freely available for redistribution

Submit your manuscript at

www.biomedcentral.com/submit 